\title{
RESPOSTA ESTOMÁTICA E PRODUÇÃO DE MATÉRIA SECA EM PLANTAS JOVENS DE AROEIRA SUBMETIDAS A DIFERENTES REGIMES HÍDRICOS ${ }^{1}$
}

\author{
Maria Alice Vasconcelos da Silva ${ }^{2}$, Rejane Jurema Mansur Custódio Nogueira ${ }^{3}$, Antônio Fernando Morais \\ de Oliveira ${ }^{4}$ Venézio Felipe dos Santos $^{5}$
}

\begin{abstract}
RESUMO - Este trabalho objetivou avaliar a transpiração, resistência difusiva do vapor d'água, a produção de matéria seca e alocação de biomassa em plantas jovens de aroeira submetidas a déficit hídrico. Foram utilizadas mudas de Schinus terebinthifolius Raddi com três meses de idade, cultivadas em vasos de polietileno contendo $5,5 \mathrm{~kg}$ de terriço vegetal. O delineamento experimental foi inteiramente casualizado com quatro tratamentos hídricos ( $100 \%$ da capacidade de campo (CC), $75 \%$ CC, $50 \%$ CC e $25 \%$ CC) e quatro repetições. Avaliaramse a transpiração e a resistência difusiva às $12 \mathrm{~h}$ em intervalos de seis dias, durante 72 dias de déficit hídrico. No final do experimento, determinaram-se a matéria seca das folhas (MSF), caules (MSC) e raízes (MSR), a alocação de biomassa de diversos órgãos e a razão raiz/parte aérea $(\mathrm{R} / \mathrm{Pa})$. O fechamento estomático ocorreu nas plantas submetidas a $25 \% \mathrm{CC}$, aos 11 dias depois da aplicação dos tratamentos. Nessa ocasião, essas mesmas plantas foram regadas até $100 \%$ CC. As regas foram suspensas até as plantas atingirem novamente $25 \%$ CC e serem mantidas nesse tratamento até o final do período experimental, não havendo mais fechamento estomático no horário estudado. A produção de matéria seca aumentou nas plantas cultivadas com $75 \%$ CC, em comparação com os demais tratamentos hídricos. Não houve diferença significativa entre os tratamentos para alocação de biomassa nos diversos órgãos. Os resultados indicam que a aroeira é moderadamente tolerante ao déficit hídrico na fase inicial do desenvolvimento.
\end{abstract}

Palavras-chave: Schinus terebinthifolius, alocação de biomassa e transpiração.

\section{STOMATAL RESPONSES AND DRY MATTER YIELD IN YOUNG AROEIRA PLANTS SUBMITTED TO DIFFERENT WATER LEVELS}

\begin{abstract}
This work aimed to evaluate gas exchange, dry matter yield and biomass allocation in young aroeira plants submitted to water deficit. Three month-old seedlings of Schinus terebinthifolius Raddi were cultivated in containers containing $5.5 \mathrm{~kg}$ of soil. A completely randomized experimental design was used, with four water treatments $(100 \%, 75 \%, 50 \%$ and $25 \%$ to field capacity-FC), with four replicates. Transpiration and diffusive resistance were evaluated at midday every seven days for 72 days. At the end of the experimental period, leaf (LDM), stem (SDM) and root dry matter (RDM) were all determined, biomass allocation of all the organs of the plants and root to shoot ratio $(R / S h)$ were also determined. Stomatal closure was observed in plants under $25 \%$ FC eleven days after water treatments. At this time, plants were watered once until 100\% FC and watering was suspended until the plants reached $25 \%$ FC again until the end of the experimental
\end{abstract}

\footnotetext{
${ }^{1}$ Recebido em 23.01.2007 e aceito para publicação em 20.02.2008.

${ }^{2}$ ProgramadePós-Graduaçãoem Ciências Florestais da UniversidadeFederal Rural dePernambuco(UFRPE).E-mail:<marriealice@hotmail.com>.

${ }^{3}$ Departamento de Biologia da UFRPE. E-mail: <rjmansur@terra.com.br>.

${ }^{4}$ Departamento de Botânica da UFRPE. E-mail: <afmoliveira@gmail.com>.

${ }^{5}$ Empresa Pernambucana de Pesquisa Agropecuária. E-mail: <venezio@ipa.br>.
} 
period and did not show stomatal closure again. After being re-watered, plants were maintained at $25 \%$ $F C$, recovering stomatal aperture, which remained open until the end of the experimental period during this evaluation time. Plants under $75 \%$ FC produced more dry matter than those in the other treatments. Significant differences among treatments in biomass allocation were not observed. These results suggest that aroeira plant is moderately drought-tolerant at the young stage of development.

Keywords: Schinus terebinthifolius, biomass allocation and transpiration.

\section{INTRODUÇÃO}

A Schinus terebinthifolius Raddi é uma espécie pertencente à família Anacardiaceae, popularmente chamada de aroeira, ou aroeira-vermelha. É originada da América do Sul, precisamente do Brasil, Paraguai, Uruguaie Argentina. No Brasil, a sua ocorrência vai desde Pernambuco até o Rio Grande do Sul (DEGÁSPARI, 2004).

Essa árvore de porte pequeno pode atingir de 5 a $10 \mathrm{~m}$ de altura e apresenta o caule com $30-60 \mathrm{~cm}$ de diâmetro. Suas flores são pequenas, agrupadas em inflorescência, melíferas, com cor amarelo-pálida a branca. O período de florescimento ocorre nos meses de setembro a janeiro, e a sua frutificação vai de janeiro a julho (LORENZI, 2002). A aroeira apresenta grande importância medicinal, por ser usada com fins terapêuticos, pois contém propriedades medicinais no combate a febre, lesões, úlceras de pele e mucosas, inflamações do útero, do sistema digestivo (gastrite, atonia gástrica, diarréia) e do sistema urinário (DEGÁSPARI et al., 2005). Além disso, a sua madeira é utilizada para moirões, esteios, lenha e carvão (LORENZI, 2002).

A aroeira é classificada como espécie pioneira e apresenta ampla distribuição geográfica, pois possui imensa plasticidade ecológica. Por essa razão, é facilmente vista em toda faixa litorânea do país, próxima a rios, córregos e várzeas úmidas de formações secundárias. Contudo, também crescem em dunas, em terrenos secos, pobres e pedregosos. Habita várias formações vegetais, sobrevivendo até seis meses sob deficiência hídrica moderada no solo (LENZI e ORTH, 2004) e sendo indicada para a recuperação de áreas degradadas (DEGÁSPARI et al., 2005).

Os efeitos da seca sobre o desenvolvimento dos vegetais dependem da intensidade, da duração do estresse e da fenologia e genética da planta, podendo provocar diversas alterações morfofisiológicas, como diminuição da turgescência e redução no tamanho das folhas, desenvolvimento de um sistema radicular mais profundo e fechamento dos estômatos nos horários mais quentes do dia (PIMENTEL, 2004).

As plantas que habitam regiões de clima predominantemente semi-árido respondem ao déficit hídrico de diversas formas para se ajustarem às condições de estresse, com o desenvolvimento de estratégias classificadas como mecanismos de escape ou de tolerância à seca (ARAÚJO, 2005). O primeiro consiste em escapar à seca através de um ciclo de vida mais curto concentrado no único período chuvoso e na formação de um banco de sementes; o segundo pode ocorrer de duas maneiras, as plantas podem tolerar a seca reduzindo o potencial hídrico interno das suas células, através do acúmulo de solutos compatíveis que favorecem a absorção de água pelo solo, ou fechar os estômatos nos horários de maior demanda evaporativa para manter o status hídrico (TURNER e BEGG, 1978; NOGUEIRA et al., 2005).

A primeira linha de defesa ao déficit hídrico é o fechamento estomático, uma vez que a resistência difusiva ao vapor de água reduz a transpiração. No entanto, se essa situação se estende acarretará prejuízo à capacidade fotossintética, uma vez que o suprimento de $\mathrm{CO}_{2}$ se torna reduzido (NOGUEIRA et al., 1998b; NOGUEIRA e SILVA, 2002), sobretudo no rendimento da produção de matéria seca (LARCHER, 2004).

Diversos autores têm pesquisado os efeitos do déficit hídrico sobre as trocas gasosas em espécies florestais (NOGUEIRA et al., 1998c, 2000; NOGUEIRA e BARBOSA, 2000; NOGUEIRA e SILVA, 2002; SILVA et al., 2003) e a produção de matéria seca (BARBOSA et al., 2000; SILVA e NOGUEIRA, 2003; FIGUEIRÔA et al., 2004). No entanto, os trabalhos desenvolvidos com plantas de aroeira sobre esse tema ainda são escassos, carecendo de informações. Dessa forma, este trabalho objetivou avaliar os efeitos do déficit hídrico sobre as trocas gasosas, a produção de matéria seca e a alocação de biomassa em plantas jovens de aroeira. 


\section{MATERIAL E MÉTODOS}

O experimento foi realizado em casa de vegetação do Laboratório de Fisiologia Vegetal do Departamento de Biologia da Universidade Federal Rural de Pernambuco (UFRPE), no período de novembro de 2005 a fevereiro de 2006.

Para a condução deste experimento, foram utilizadas mudas de aroeira (Schinus terebinthifolius Raddi) com aproximadamente três meses de idade, obtidas por propagação sexuada, originadas da mesma planta-matriz e cedidas pelo Departamento de Biologia da UFRPE. Quando as mudas atingiram cerca de $30 \mathrm{~cm}$ de altura, elas foram transferidas para vasos de polietileno contendo $5,5 \mathrm{~kg}$ de terriço vegetal.

O delineamento experimental utilizado foi inteiramente casualizado, com quatro tratamentos hídricos ( $100 \%$ da capacidade de pote (CC), $75 \%$ CC, $50 \%$ CC e $25 \%$ CC) com quatro repetições. A capacidade de pote foi calculada segundo a metodologia descrita por Souza et al. (2000), com uma modificação (água adicionada pela superfície do substrato). O conteúdo da água do vaso foi estabelecido em três repetições, por meio da determinação da quantidade de água retida no solo seco ao ar após sofrer saturação e conseqüente ação da gravidade até a completa drenagem.

Durante o período de aclimatação de 15 dias, todos os recipientes foram mantidos na capacidade de pote (100\% CC). Após esse período, procedeu-se à diferenciação dos tratamentos hídricos. As superfícies dos vasos foram cobertas com um plástico opaco, com o intuito de evitar a perda da água por evaporação do substrato. Para manter os níveis de água estabelecidos, a água perdida por transpiração foi reposta diariamente através da pesagem dos vasos, de acordo com cada nível de água.

As avaliações da transpiração (E) e da resistência difusiva ( $\mathrm{Rs}$ ) foram realizadas diariamente em folhas completamente expandidas, localizadas no terço médio das plantas, sempre às $12 \mathrm{~h}$, horário de maior demanda evaporativa verificada através de um curso diário antes da aplicação dos tratamentos hídricos (Figura 1). Para tanto, utilizou-se um Porômetro de Equilíbrio Dinâmico da LICOR (modelo LI - 1600). De forma simultânea, foram registradas a umidade relativa do ar (UR), a temperatura do ar $\left(\mathrm{T}_{\mathrm{ar}}\right)$ e a radiação fotossinteticamente ativa (PAR), por meio de um sensor quântico acoplado ao mesmo Porômetro. O déficit de pressão de vapor (DPV) foi calculado a partir dos dados de Tar e da UR.
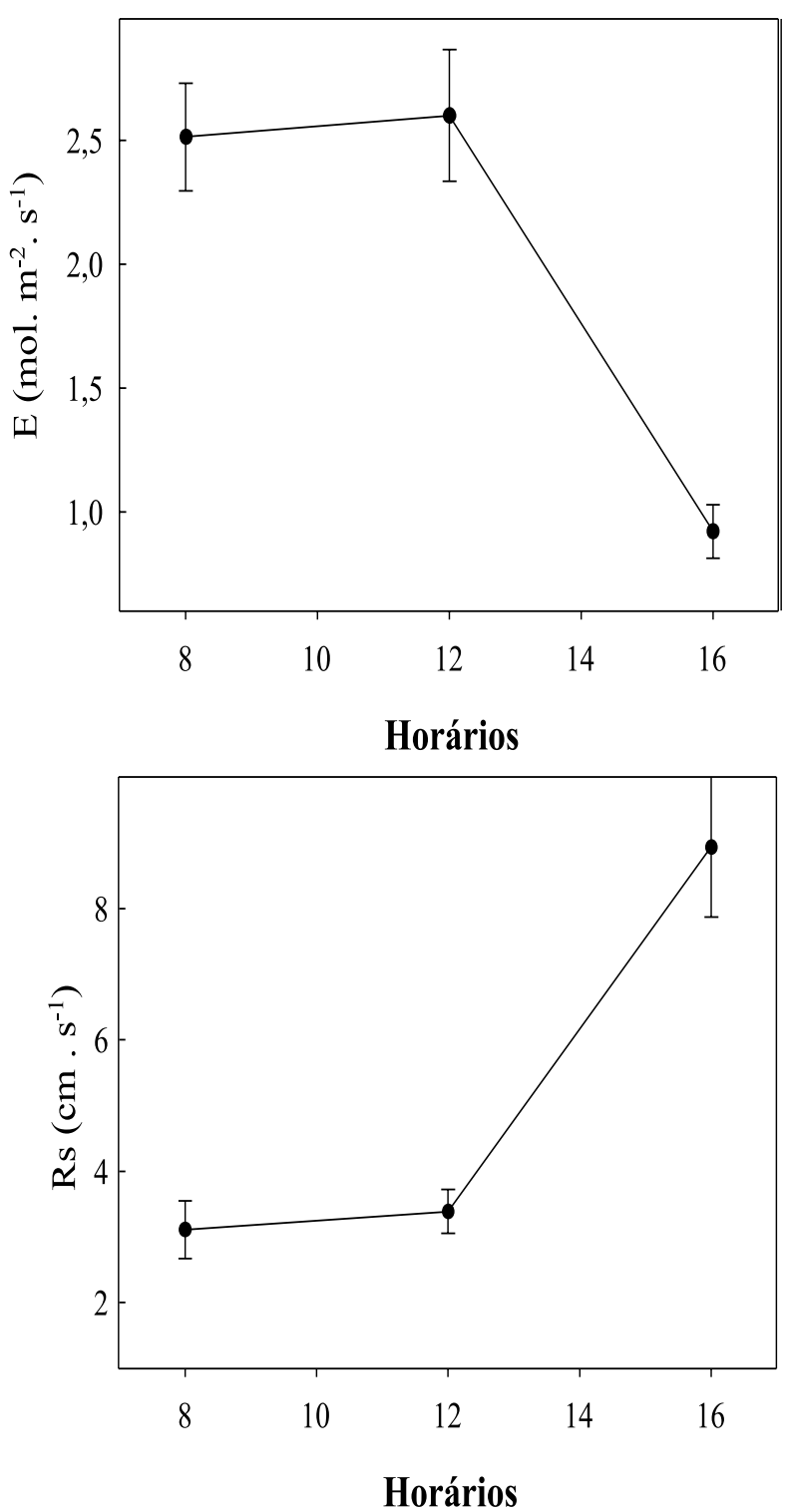

Figura 1 - Curso diário da transpiração (E) e da resistência difusiva (Rs) em plantas jovens de aroeira (Schinus terebinthifolius Raddi) cultivadas em solo na capacidade de campo em casa de vegetação.

Figure 1 -Daily course of transpiration $(T)$ and diffusive resistance $(D R)$ in young aroeira plants (Schinus terebinthifolius Raddi) cultivated in soil field capacity under greenhouse conditions.

R. Árvore, Viçosa-MG, v.32, n.2, p.335-344, 2008 
No final do experimento, as plantas foram colhidas e separadas em folhas, caules e raízes. As raízes foram lavadas em água corrente para retirar todos os resíduos de solo, secas em papel absorvente e acondicionadas em sacos de papel e levadas à estufa de circulação de ar forçada a $65^{\circ} \mathrm{C}$ até atingir peso constante. A determinação do peso da matéria seca das folhas (MSF), dos caules (MSC), das raízes (MSR) e da matéria seca total (MST). Com os dados da matéria seca, calculou-se a alocação de biomassa para as folhas (ABF), caules (ABC) e raízes (ABR) e a relação raiz/parte aérea $(\mathrm{R} / \mathrm{Pa})$, segundo Benincasa (1988).

Os dados foram submetidos à análise descritiva e à análise de variância (ANOVA), sendo as médias comparadas pelo teste de Tukey a 5\% de probabilidade. Foram determinadas correlações lineares simples, para estimar o grau de relação entre as variáveis fisiológicas e climáticas, utilizandose o programa STATISTIX 7.0.

\section{RESULTADOS E DISCUSSÃO}

Durante o período experimental, a Tar variou em média de $27,4^{\circ} \mathrm{C}$ a $33,4^{\circ} \mathrm{C}$, a UR de $32,5 \%$ a $53,5 \%$, a PAR de $108,4 \mu \mathrm{mol} . \mathrm{m}^{-2} \cdot \mathrm{s}^{-1} \mathrm{a} 466,8 \mu \mathrm{mol} . \mathrm{m}^{-2} . \mathrm{s}^{-1}$ e o DPV em média de 1,7 kPa a 3,4 kPa (Quadro 1). Essa variação se deve à presença de muitas nuvens no céu durante o período experimental.
Foram observadas variações nas taxas de transpiração das plantas ao longo do período experimental. Essas variações se devem parte às condições climáticas, como Tar, UR e PAR (Figura 3) e parte às diferentes saturações hídricas estabelecidas nos tratamentos. O déficit hídrico mais severo (25\% $\mathrm{CC})$ reduziu a transpiração (E) das plantas estressadas. Foi observado fechamento estomático aos 11 dias de tratamento hídrico nas plantas submetidas a $25 \%$ CC $\left(0,46 \mathrm{mmol} \cdot \mathrm{m}^{-2} \cdot \mathrm{s}^{-1}\right)$, as quais recuperaram a transpiração após serem regadas até atingir $100 \%$ CC $\left(5,5 \mathrm{mmol} \cdot \mathrm{m}^{-2} \cdot \mathrm{s}^{-1}\right)$. As plantas foram, então, mantidas sob $25 \%$ CC, e os estômatos permaneceram abertos até o final do período experimental no horário avaliado, ou seja, por mais 60 dias mantidos sob $25 \%$ CC (Figura 3). A literatura reporta que a deficiência hídrica afeta, primeiramente, o aparato estomático, provocando o fechamento dos estômatos, porém, quando as plantas passam por ciclos de seca, elas podem desenvolver certa resistência à falta de água, mantendo a transpiração por períodos mais longos (LARCHER, 2004). Os resultados encontrados neste trabalho suportam essa afirmativa.

Nogueira et al. (1998c), estudando três espécies lenhosas da caatinga, observaram que a transpiração foi reduzida após 20 dias de suspensão de rega. O mesmo foi observado em plantas jovens de craibeira, tamboril e sabiá cultivadas sob $50 \%$ CC (SILVA et al., 2003).

Quadro 1 - Valores médios da temperatura do ar (Tar), umidade relativa do ar (UR), radiação fotossinteticamente ativa (PAR) e déficit de pressão de vapor (DPV) durante o período experimental. Médias de quatro repetições \pm desviopadrão

Table 1 - Means values of air temperature (Tar), relative humidity of the air $(R H)$, photosynthetically-active radiation $(P A R)$ and vapor pressure deficit (VPD) during the experimental period. Means \pm standard-deviation of four replicates

\begin{tabular}{cccc}
\hline Dias após tratamento & Tar $\left({ }^{\circ} \mathrm{C}\right)$ & UR $(\%)$ & PAR $\left(\mathrm{mmol} \cdot \mathrm{m}^{-2} \cdot \mathrm{s}^{-1}\right)$ \\
\hline 4 & $32,8 \pm 1,3$ & $34,2 \pm 2,9$ & $395,9 \pm 99,9$ \\
11 & $33,0 \pm 0,8$ & $32,5 \pm 3,3$ & $409,0 \pm 204,9$ \\
18 & $32,3 \pm 1,1$ & $37,3 \pm 4,4$ & $466,8 \pm 110,6$ \\
24 & $27,4 \pm 1,1$ & $53,5 \pm 5,1$ & $108,4 \pm 37,2$ \\
30 & $31,6 \pm 1,3$ & $37,1 \pm 3,2$ & $231,0 \pm 167,5$ \\
36 & $32,3 \pm 0,6$ & $33,4 \pm 2,1$ & $334,1 \pm 197,7$ \\
42 & $31,8 \pm 1,0$ & $37,6 \pm 3,3$ & $276,2 \pm 145,2$ \\
48 & $32,3 \pm 0,9$ & $33,7 \pm 3,1$ & $447,5 \pm 87,6$ \\
54 & $32,8 \pm 0,4$ & $33,9 \pm 4,0$ & $310,1 \pm 230,0$ \\
60 & $32,5 \pm 0,8$ & $34,8 \pm 3,3$ & $409,4 \pm 162,7$ \\
66 & $33,4 \pm 0,9$ & $35,8 \pm 3,0$ & $344,2 \pm 186,8$ \\
72 & $33,1 \pm 1,0$ & $33,6 \pm 4,6$ & $452,5 \pm 248,9$ \\
\hline
\end{tabular}


A transpiração (E) correlacionou-se positivamente com Tar e com DPV e, de forma negativa, com a UR e Rs em todos os níveis de água. Houve correlação com a PAR apenas nos tratamentos de $50 \%$ CC e $25 \%$ CC. Isso indica que os fatores climáticos também
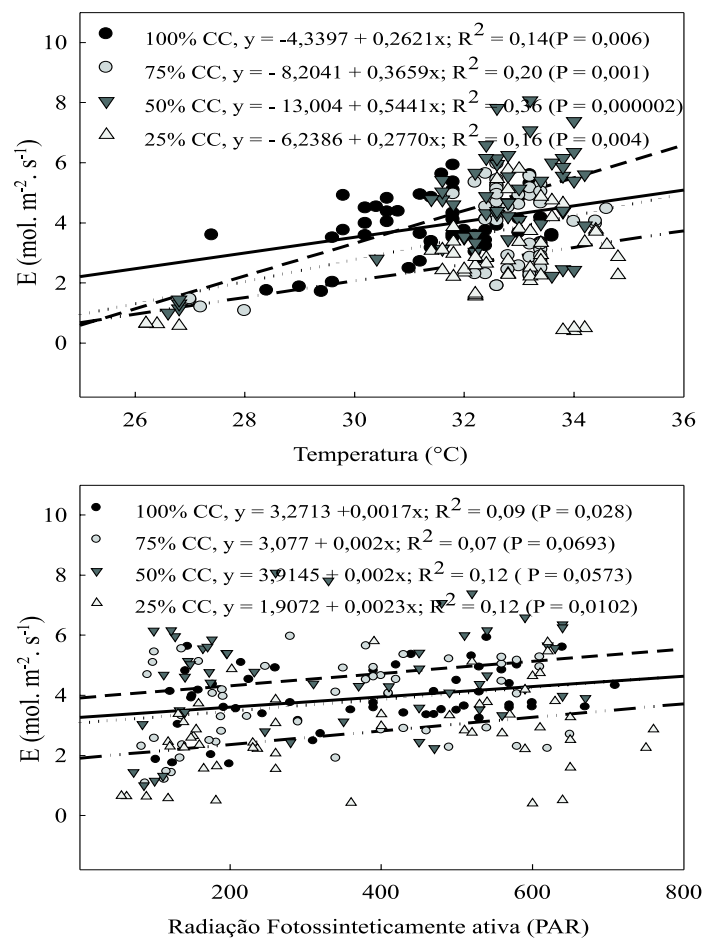

influenciaram a perda de água por transpiração, mesmo nas plantas submetidas a déficit hídrico (Figura 2). Outros pesquisadores observaram influência da Tar, PAR, UR e DPV no comportamento estomático de plantas Schinopsis brasiliensis (NOGUEIRA et al., 2002).
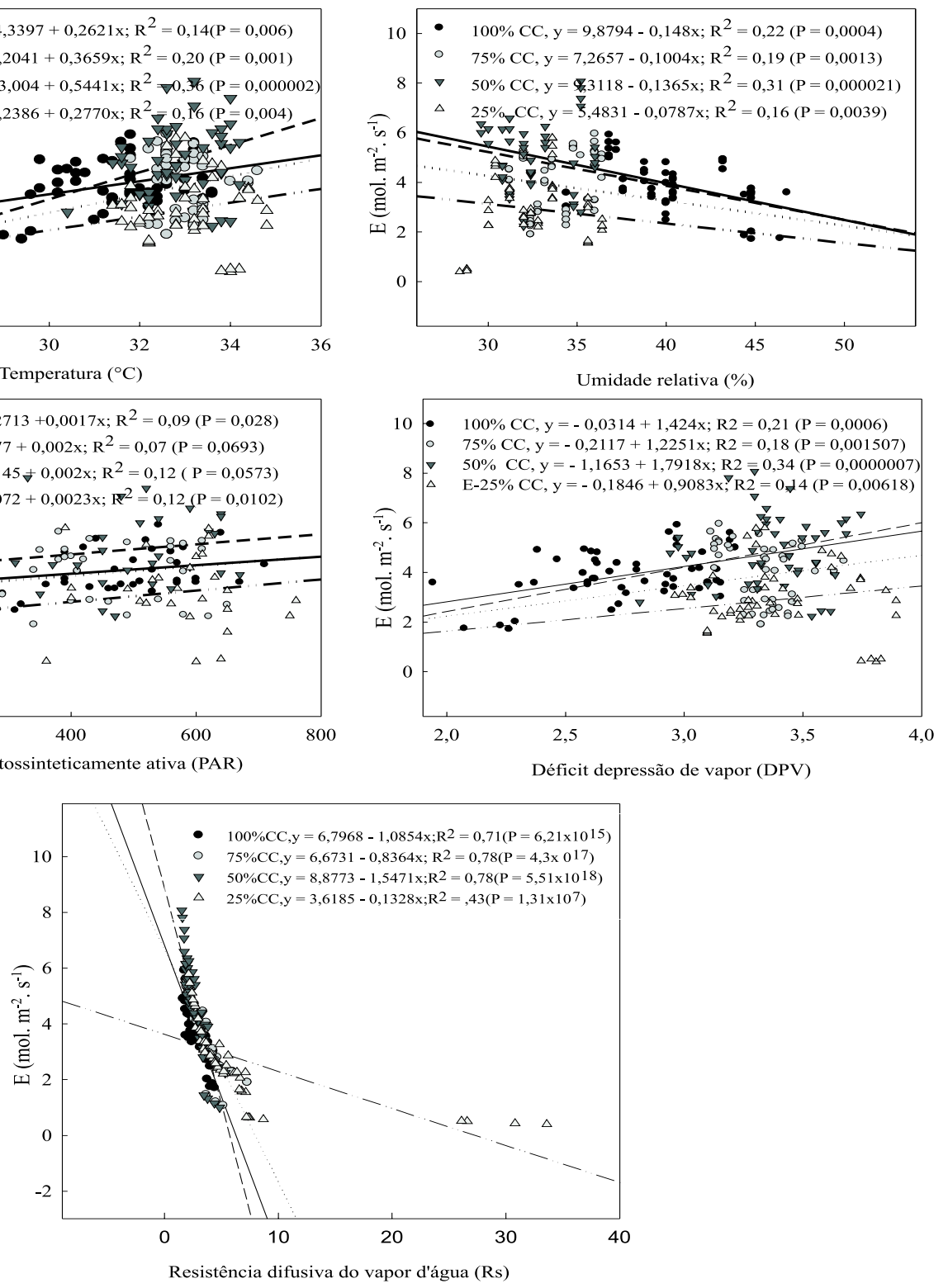

Figura 2 - Regressão linear das correlações entre a resistência difusiva (Rs), a transpiração (E), a temperatura do ar (Tar), a umidade relativa do ar (UR), a radiação fotossinteticamente ativa (PAR) e os diferentes regimes hídricos em plantas jovens de aroeira (Schinus terebinthifolius Raddi).

Figure 2 -Linear regression of the correlations among diffusive resistance (DR), transpiration (T), air temperature (AT), relative air humidity $(R A H)$ and photosynthetically-active radiation $(P A R)$ and the different water levels in young aroeira plants (Schinus terebinthifoliuks Raddi). 
O nível de água de $25 \%$ CC provocou aumento significativo na Rs das plantas em relação às plantascontrole $(100 \% \mathrm{CC})$, com os maiores valores observados após 11 dias de tratamento hídrico $\left(29,3 \mathrm{s.cm} \mathrm{cm}^{-1}\right)$, como observado na Figura 3. Aos 11 dias após o início do estabelecimento dos diferentes níveis de água no solo, onde foi detectada a maior Rs em $25 \% \mathrm{CC}$, coincidentemente foi o dia com elevada Tar e mais baixa UR, alto PAR e, conseqüentemente, o maior DPV de todo o período experimental. Diante disso, o fechamento estomático nesse dia pode ter ocorrido em função das condições climáticas adversas nesse dia. Após as plantas receberem água no nível de $100 \%$ CC, a resistência diminuiu consideravelmente e manteve-se baixa, à semelhança dos demais tratamentos, ao longo dos 60 dias subsequientes (Figura 3). As plantas que reduzem a abertura dos estômatos em situação de déficit hídrico são mais conservativas no uso da água (CHAVES et al., 2004).

Silva et al. (2004), estudando o comportamento fisiológico de nove espécies da caatinga, observaram variações no comportamento estomático entre elas. A maioria das espécies estudadas elevou a Rs nos horários mais quentes, permanecendo assim até o final do dia, enquanto outras, como Croton campestris e Caesalpinia pyramidalis, reduziram os valores de Rs às $16 \mathrm{~h}$. Neste trabalho, observou-se comportamento semelhante aos da maioria das plantas verificadas por Silva et al. (2004), que apresentaram elevação da Rs nos horários mais quentes do dia, com permanência até o final do dia (Figura 1). Em contraste, Chaves et al. (2004), avaliando clones de eucalipto plenamente irrigados e sob déficit hídrico, não observaram diferença significativa entre os tratamentos hídricos na condutância estomática (inverso da Rs). O comportamento observado na aroeira, fechando os estômatos quando cultivada com $25 \%$ CC e mantendoos abertos após a reirrigação, evidencia um possível ajustamento da espécie à condição de seca.

A resistência difusiva (Rs) não apresentou correlação com a Tar, UR, PAR e DPV, demonstrando que a água foi o fator que realmente influenciou o fechamento estomático (Figura 2). Silva et al. (2003) também observaram que a Rs de plantas jovens de tamboril (Enterolobium contortisiliquum) e craibeira (Tabebuia aurea), submetidas a dois regimes hídricos, não apresentaram correlação com UR, PAR e DPV.

O estresse hídrico provocou maior acúmulo na MSF das plantas submetidas a $75 \%$ CC $\left(9,72\right.$ g.planta $\left.{ }^{-1}\right)$, representando um acréscimo de $60 \%$ em relação ao tratamento de $100 \%$ CC (5,73 g.planta $\left.{ }^{-1}\right)$ (Figura 4). Porém, não houve diferença significativa entre os demais tratamentos hídricos (Figura 4). Quanto a MSC, apenas o tratamento de $75 \%$ CC $\left(11,10\right.$ g.planta $\left.{ }^{-1}\right)$ diferiu significativamente do tratamento $25 \%$ CC (6,10 g.planta ${ }^{-}$ ${ }^{1}$ ), como mostrado na Figura 4, não havendo diferença entre os demais tratamentos. Na MSR e MST, não houve diferença significativa entre os níveis de água no solo (Figura 4). No entanto, de forma geral observou-se tendência nas plantas do tratamento de $75 \% \mathrm{CC}$ de produzir maior quantidade de matéria seca do que os demais tratamentos hídricos (Figura 4).

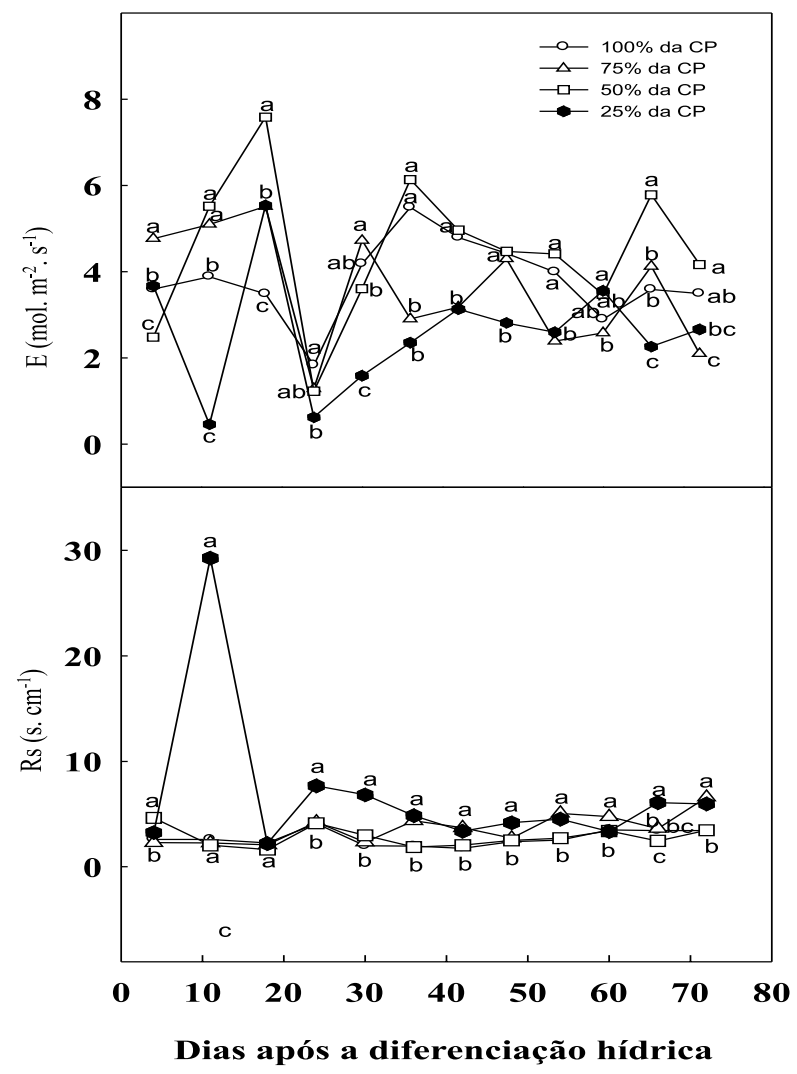

Figura 3 - Transpiração (E) e resistência difusiva (Rs) ao longo do tempo, em plantas jovens de aroeira (Schinus terebinthifolius Raddi) sob diferentes regimes hídricos. Valores seguidos de mesmas letras entre os tratamentos hídricos não diferem entre si, pelo teste de Tukey a $5 \%$ de probabilidade.

Figure 3 - Transpiration (T) and diffusive resistance (DR) over time in young aroeira plants (Schinus terebinthifoliuks Raddi) under different water levels. Values followed by the same letters among treatments do not differ significantly according to Tukey's multiple range test at $P<0.05$. 


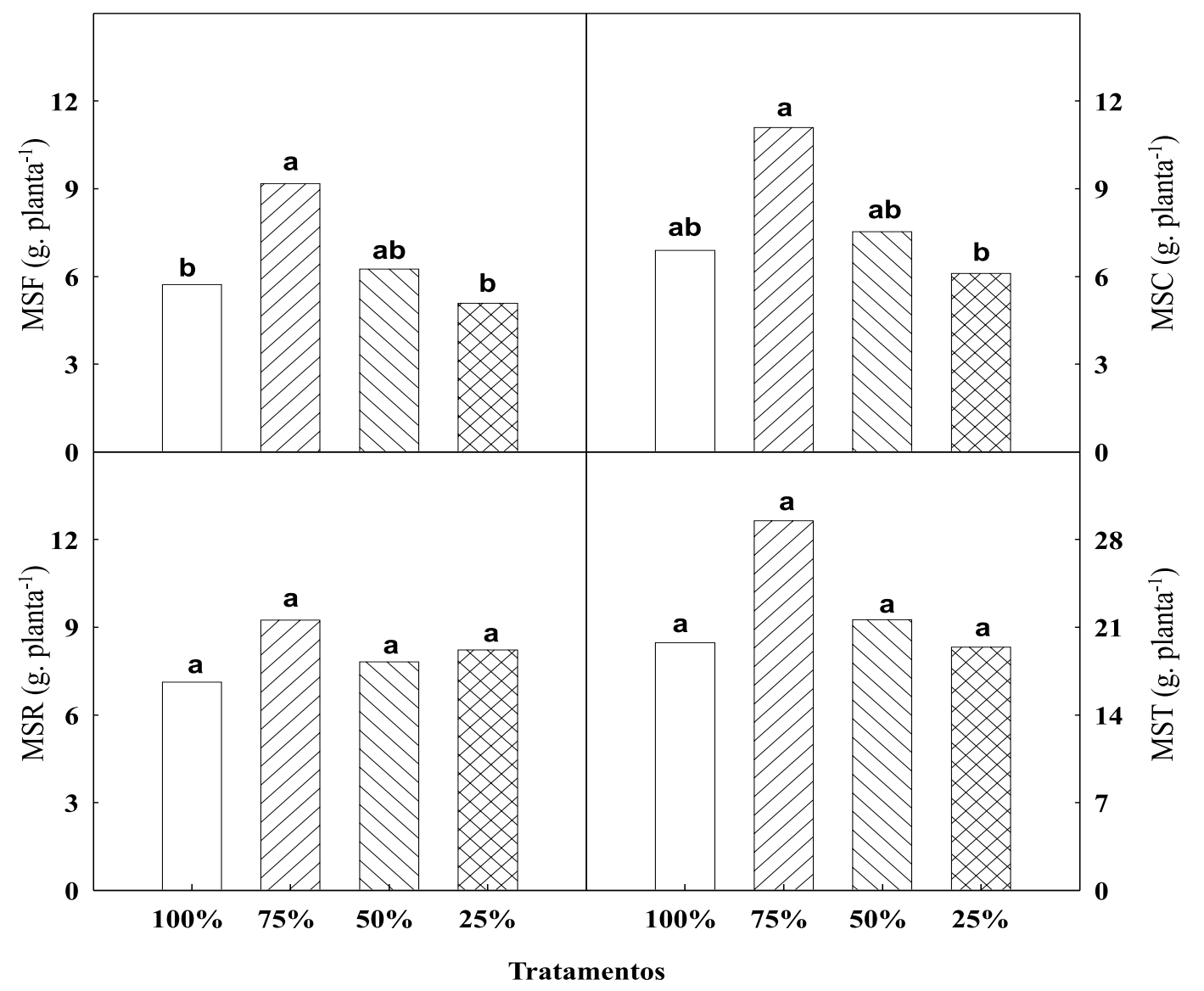

Figura 4 - Matéria seca das folhas (MSF), dos caules (MSC) e das raízes (MSR) e matéria seca total (MST) de plantas jovens de aroeira (Schinus terebinthifolius Raddi) sob diferentes regimes hídricos. Tratamentos: 100\% CC (capacidade de pote) $\square, 75 \% \mathrm{CC} \square / \lambda, 50 \% \mathrm{CC} \triangle \backslash$ e 25\% CC $\square \times \square$. Médias seguidas por letras iguais não diferem estatisticamente entre si, pelo teste de Tukey $(\mathrm{P}<0,05)$.

Figure 4-Leaves (LDM), stems (SDM), roots (RDM) and total dry masses (TDM) in young aroeira plants (Schinus terebinthifolius Raddi) under different water levels. Treatments: $100 \%$ field capacity (FC) $\square, 75 \%$ FC $\square \backslash, 50 \%$ FC $\square \backslash$ and $25 \%$ FC $\square$. Values followed by the same letters do not differ significantly according to Tukey's multiple range test at $P<0.05$.

Oliveira (2000), estudando o crescimento de mudas de gravioleira sob déficit hídrico, observou maior produção de matéria seca na raiz $(8,44 \mathrm{~g}$.planta-1) e na matéria seca total $(30,95$ g.planta-1) em plantas de gravioleira sob $75 \%$ CC. No entanto, Santiago (2000), avaliando o crescimento de plantas jovens de sabiá (Mimosa caesalpiniifolia Benth), verificou que o déficit hídrico provocou redução tanto na matéria seca das folhas quanto na matéria seca do caule. Em adição, Leite (2003) verificou que o déficit hídrico não reduziu a MSR em clones de cajueiro anão-precoce, variedade EMBRAPA-50.
A resposta das plantas ao déficit hídrico depende dos seus estádios fenológico e genético e da magnitude e intensidade do estresse. Um déficit hídrico semimoderado pode beneficiar o desenvolvimento das plantas, favorecendo o crescimento e produção de biomassa (PIMENTEL, 2004), como observado neste trabalho, nas plantas do tratamento $75 \% \mathrm{CC}$.

Também não se observou diferença significativa entre os tratamentos para a alocação de biomassa das folhas (ABF), dos caules (ABC) e das raízes (ABF) e a razão raiz/parte aérea $(\mathrm{R} / \mathrm{Pa})$ (Figura 5).

R. Árvore, Viçosa-MG, v.32, n.2, p.335-344, 2008 


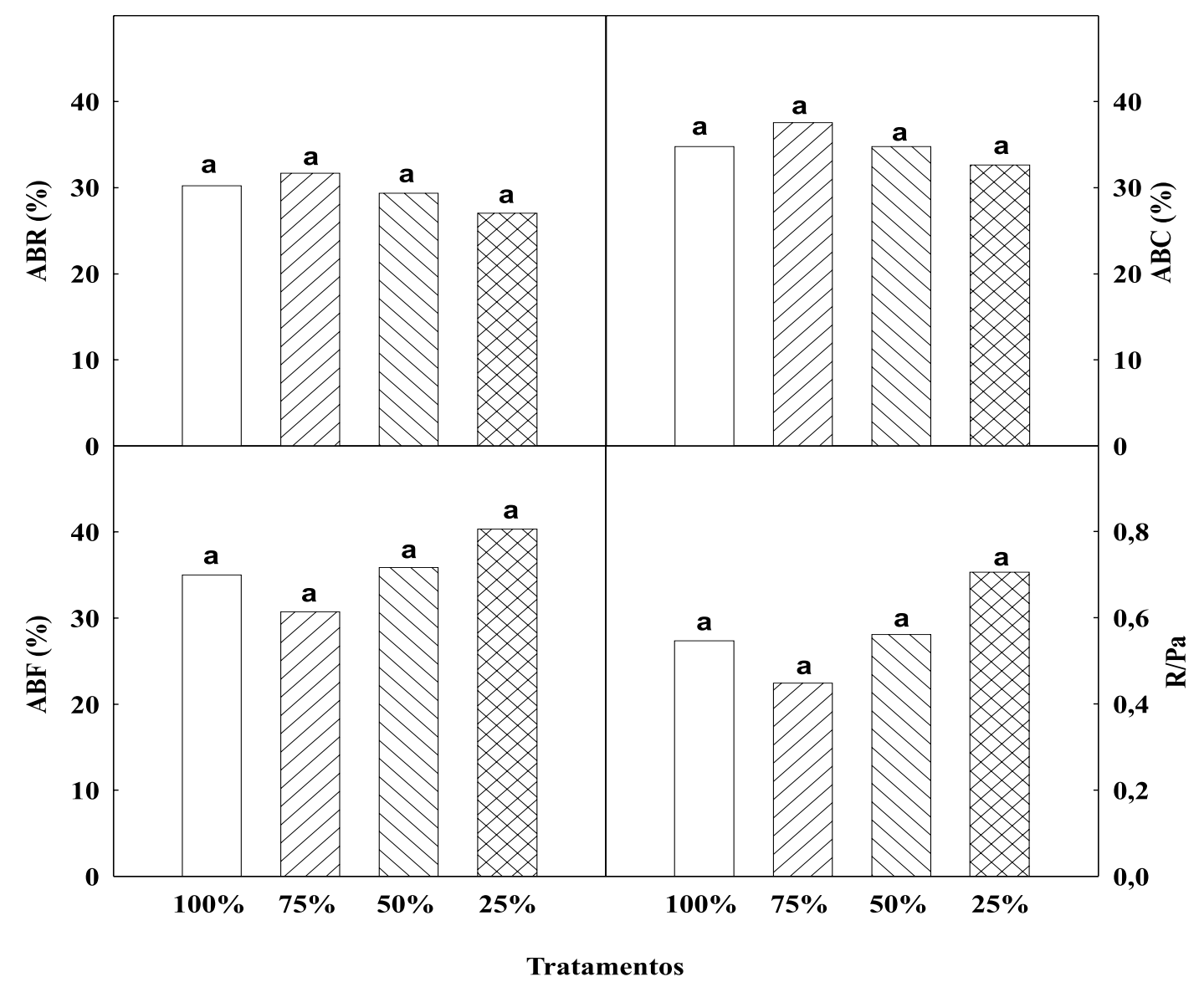

Figura 5 - Alocação de biomassa das folhas (ABF), dos caules (ABC) e das raízes (ABR) e razão raiz/parte aérea (R/Pa) de plantas jovens de aroeira (Schinus terebinthifolius Raddi) sob diferentes regimes hídricos. Tratamentos: $100 \%$

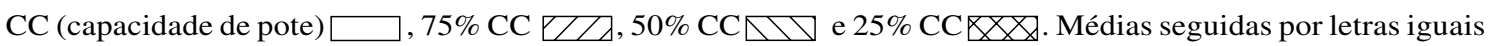
não diferem estatisticamente entre si, pelo teste de Tukey $(\mathrm{P}<0,05)$.

Figure 5 - Biomass allocation to leaves $(B A L)$, stems $(B A S)$ and roots (BAR) and root to shoot ratio (R/Sh) in young aroeira plants (Schinus terebinthifoliuks Raddi) under different water levels. Treatments: $100 \%$ field capacity $(F C) \square$, $75 \%$ FCE according to Tukey's multiple range test at $P<0.05$.

A literatura reporta que plantas submetidas a déficit hídrico severo tende a investir mais no alongamento da raiz do que na parte aérea, para absorver água das zonas mais profundas do solo (BARROS e BARBOSA, 1995; BARBOSA et al., 2000; SILVA e NOGUEIRA, 2003). Isso foi verificado por Figueirôa et al. (2004), quando estudaram os efeitos do estresse hídrico no crescimento de Myracrodruon urundeuva. Esses autores observaram que houve maior alocação de biomassa para a raiz nas plantas sob $25 \%$ CC $(43,6 \%)$ em relação aos tratamentos de $75 \%$ CC (22\%) e $50 \%$ CC (18\%), aumentando a R/Pa.
O mesmo foi observado por Villagra e Cavagnaro (2006) em plantas de Prosopis argentina e Prosopis alpataco sob déficit hídrico. Esse comportamento, no entanto, não foi observado neste trabalho, uma vez que não houve reduções significativas na produção de matéria seca dos diversos órgãos.

Os resultados deste trabalho permitem inferir que a aroeira é tolerante a baixos níveis de água no solo, uma vez que na fase inicial do desenvolvimento mantiveram estômatos abertos mesmo sob significativa limitação de água. 


\section{CONCLUSÃO}

Plantas jovens de aroeira crescem melhor quando cultivadas com suprimento hídrico de $75 \%$ da capacidade de campo, podendo ser cultivadas em níveis de água de até $50 \%$ CC, sem apresentar modificações morfológicas e fisiológicas significativas.

A aroeira é moderadamente tolerante a baixos níveis de água no solo.

\section{REFERÊNCIAS}

ARAÚJO, E. L. Estresses abióticos e bióticos como forças, modeladoras da dinâmica de populações vegetais da caatinga. In: NOGUEIRA, R. J. M. C. et al. Estresses ambientais: danos e benefícios em plantas. Recife: Universidade Federal Rural de Pernambuco, 2005. 500p.

BARBOSA, D. C.; NOGUEIRA, R. J. M. C.; MELO FILHO, P. A. Comparative studies of growth in three species of "caatinga" sumetted to water stress. Phyton, v.69, n.1, p.45-50. 2000.

BARROS, L. M.; BARBOSA, D. C. A. Crescimento de Acacia farnesiana (L.) Willd em casa de vegetação. Phyton, v.57, n.2, p.179-191, 1995.

BENINCASA, M. M. P. Análise de crescimento de plantas. Jaboticabal: FUNEP, 1988. 42p.

CHAVES, J. H. et al. Seleção precoce de clones de eucalipto para ambientes com disponibilidade diferenciada de água no solo: relações hídricas de plantas em tubetes. Revista Árvore, v.28, n.3, p.333-341, 2004.

DEGÁSPARI, C. H. Propriedades antioxidantes e antimicrobianas dos frutos da aroeira (Schinus terebenthifolius Raddi). 2004. 104f. Tese (Doutorado em Tecnologia de Alimentos) Universidade Federal do Paraná, Curitiba, 2004.

DEGÁSPARI, C. H.; WASZCZYNSKYJ, N.; PRADO, M. R. M. Atividade antimicrobiana de Schinus terebenthifolius Raddi. Ciência. Agrotécnica, v.29, n.3, p.617-622, 2005.

FIGUEIRÔA, J. M.; BARBOSA, D. C.A.; SIMABUKURO, E. A. Crescimento de plantas jovens de Miracrodruon urundeuva Allemão (Anacardiaceae) sob diferentes regimes hídricos. Acta Botânica Brasílica, v.18, n.3, p.573-580, 2004.
LARCHER, W. Ecofisiologia vegetal. São

Carlos: Rima, 2004. 531p.

LEITE, F. V. A. Respostas fisiológicas do cajueiro anão-precoce (Anacardium occidentale L.) ao déficit hídrico. 2003. 73f. Dissertação (Mestrado em Botânica) Universidade Federal Rural de Pernambuco, Recife, 2003.

LENZI, M.; ORTH, A. I. Fenologia reprodutiva, morfologia e biologia floral de Schinus terebinthifolius Raddi (Anacardiaceae), em restinga da ilha de Santa Catarina, Brasil. Biotemas, v. 17, n.2, p.67-89, 2004.

LORENZI, H. Árvores brasileiras: manual de identificação e cultivo de plantas arbóreas nativas do Brasil. 4.ed. São Paulo: Instituto Plantarum, 2002. v.1. 368p.

NOGUEIRA, R. J. M. C. et al. Curso diário do potencial hídrico foliar em cinco espécies da caatinga. Revista Ecossistema. v.23, n.1, p.73-77, 1998 b.

NOGUEIRA, R. J. M. C.; BARBOSA, D. C. A.; MORAES, J. A. P. Trocas gasosas e relações hídricas em plantas jovens envasadas de três espécies da caatinga, submetidas à deficiência hídrica. $\phi$ YTON, v.62, n.1/2, p.37-46, 1998 c.

NOGUEIRA, R. J. M. C. et al. Comportamiento estomático y tensión de agua en el xilema de dos genotipos de pitanga (Eugenia uniflora L.) cultivados bajo estrés hídrico. Investigación Agraria: Producción Protección Vegetales, v.15, n.3, p.213-225, 2000.

NOGUEIRA, R. J. M. C.; BARBOSA, D. C. A. Comportamento fisiológico em plantas jovens de quatro espécies lenhosas da caatinga submetidas a dois ciclos de estresse hídrico. $\phi$ YTON, v.68, n.1, p.97-106, 2000.

NOGUEIRA, R. J. M. C.; SILVA, E. C.

Comportamento estomático em plantas jovens de Schinopsis brasiliensis Engl. cultivadas sob estresse hídrico. Iheringia, Série Botânica, v.57, n.1, p.31-38, 2002.

R. Árvore, Viçosa-MG, v.32, n.2, p.335-344, 2008 
NOGUEIRA, R. J. M. C. et al. Curso diário das perdas de vapor d'água, da temperatura e do potencial hídrico da água da folha em germoplasma de carambola (Averrhoa carambola L.). Acta Botânica Brasílica, v.16, n.2, p.217-223, 2002.

NOGUEIRA, R. J. M. C. et al. Aspectos ecofisiológicos da tolerância à seca em plantas da caatinga. In: NOGUEIRA, R. J. M. C. et al. Estresses ambientais: danos e benefícios em plantas. Recife: Universidade Federal Rural de Pernambuco, 2005.500p.

OLIVEIRA, D. V. Aspectos do crescimento da gravioleira (Annona muricata L.) sob estresse hídrico. 2000. 60f. Dissertação (Mestrado em Botânica) - Universidade Federal Rural de Pernambuco, Recife, 2000.

PIMENTEL, C. A relação da planta com a água. Seropédica: Edur, 2004. 191p.

SAntiago, A. M. P. Aspectos do crescimento do sabiá (Mimosa caesalpiniifolia Benth.) em função da disponibilidade de água no solo. 2000. $63 \mathrm{f}$. Dissertação (Mestrado em Botânica) - Universidade federal Rural de Pernambuco, Recife, 2000.
SILVA, E. C.; NOGUEIRA, R. J. M. C. Crescimento de quatro espécies lenhosas cultivadas sob estresse hídrico em casa de vegetação. Revista Ceres, v.50, n.288, p.203-217, 2003.

SILVA, E. C. et al. Comportamento estomático e potencial da água da folha em três espécies lenhosas cultivadas sob estresse hídrico.

Acta Botânica Brasílica, v. 17, n.2, p.231-246, 2003.

SOUZA, C. C. et al. Avaliação de métodos de determinação de água disponível e manejo da irrigação em terra roxa sob cultivo de algodoeiro herbáceo. Revista Brasileira de Engenharia Agrária e Ambiental, v.4, n.3, p.338-342, 2000.

TURNER, N. C.; BEGG, J. E. Responses of pasture plants to water deficits. In: WILSON, J. R. (Org.) Plant relations in pastures. Melbourne: CSIRO, 1978. p.50-66.

VILLAGRA, P. E.; CAVAGNARO, J. B. Water stress effects on the seedling growth of Prosopis argentina and Prosopis alpataco. Journal of Arid Environments, v.64. p.390-400, 2006. 